\title{
Apoyo social laboral y extralaboral en los médicos familiares que laboran para dos instituciones de salud en Guadalajara, México
}

\author{
CAROLINA ARANDA(1) y MANUEL PANDO(1)
}

\section{RESUMEN}

Es conocido que el apoyo social genera en el individuo un desarrollo óptimo emocional y satisfactorio con consecuencias positivas hacia su salud, en beneficio de su calidad de vida y de las personas que le rodean. La percepción que el sujeto tiene de que recibe apoyo, de que su red es confiable y efectiva, es un determinante importante de su estado de salud, independientemente de la condición real del sujeto.

El objetivo del trabajo fue analizar las condiciones en que se encuentra el apoyo social extralaboral y laboral y su asociación con alguna variables sociodemográficas y laborales en los médicos familiares, que laboran para dos instituciones de salud en la zona metropolitana de Guadalajara, México.

El estudio es descriptivo y transversal. La población incluye a los médicos familiares de ambas instituciones. Se les aplicó un cuestionario de datos generales y el inventario de Recursos Sociales de Díaz Veiga para evaluar el apoyo social.

Participaron 196 médicos, 73 mujeres y 123 hombres, el promedio de edad fue de 46.29 años, la mayoría de ellos son casados (79,7\%), el nivel máximo de estudios es la especialidad $(92,9 \%)$ y el 52,8\% trabaja por las mañanas. En general las redes sociales de apoyo con las que cuentan los médicos fueron "muy buenas", lo bastante como para no observarse asociación ni factores de riesgo con alguna manifestación en salud.

Se sugiere continuar con su red apoyo y, para quienes no la tienen, habrá que crearla y mantenerla adecuadamente.

Palabras clave: apoyo social, red de apoyo, médicos familiares, instituciones de salud.

\section{ABSTRACT}

LABOUR AND EXTRA-LABOUR-BASED SOCIAL SUPPORT AMONG FAMILY PHYSICIANS EMPLOYED BY TWO HEALTH CARE INSTITUTIONS IN GUADALAJARA, MEXICO.

The fact that social support generates satisfaction and an optimal emotional development in an individual, resulting in positive consequences for his/her health and providing benefits for both the quality of life of the individual and the people belonging to his/her human environment, is well known. The individual's perception of receiving support and the awareness of a reliable and effective network constitute a determining factor for his/her health status, irrespectively of his/her real condition.

(1) Departamento de Salud Pública. Universidad de Guadalajara. caranda2000@yahoo.com.mx 
The purpose of the present work was to analyse the current status of labour and extra-labourbased social support and to assess its association with any social-demographic and labour variables among family physicians employed by two health care institutions at the urban area of Guadalajara, Mexico.

The study design was cross-sectional and descriptive. The study population consisted of family physicians from both institutions. A general data survey and the Social Resources Inventory of Díaz Veiga were conducted to assess social support.

From a total of 196 physicians who were enrolled, 73 were female and 123 were male. The mean age was 46.29. Most of them were married (79.7\%). The highest degree in education attained was the medical specialty (92.9\%) and 52.8\% worked during the mornings. In general, social support networks available to the physicians were ranked as "very good". The latter was decisive enough as to explain the lack of associations with or risk factors for any manifestation of disease. The recommendation is to maintain the available support network, and for those lacking one, to create a support network and to adequately maintain it.

Keywords: social support, support network, family physicians, health care institutions.

\section{INTRODUCCIÓN}

El concepto sobre apoyo social ha sido definido de diferentes maneras y perspectivas. Algunos autores lo conceptualizan como la disponibilidad de ayuda proveniente de otras personas $^{1}$, o como el grado en que las necesidades sociales básicas se satisfacen a través de la relación recíproca con los otros ${ }^{2}$, como aquellas acciones que se realizan entre las personas ${ }^{3-5}$, o como la disponibilidad de dar información y ofrecer recursos para disminuir los procesos estresantes y afrontar de manera adecuada los acontecimientos.

Podemos enfatizar que en todos estos conceptos se habla de esa interrelación que se da entre las personas, en la cual no importa la cantidad de personas que puede ofrecer apoyo sino la calidad de éste. Sin embargo, no podemos referirnos sólo al concepto de apoyo social si no conceptualizamos a las "redes sociales de apoyo", ya que para esa interrelación entre las personas se necesita de, por lo menos, la presencia de alguien más para decir que se cuenta con una red de apoyo.

La red social de apoyo ya sea formal (escuela, institución, empresa, etc) o informal (brindada principalmente por la familia) es el conjunto de personas que intercambia de manera continua y recíproca una serie de acciones y/o conductas que también se relaciona entre sí al demostrarse cariño; interés; escucharse; prestarse objetos materiales; ayuda económica y moral a alguien; dar y sentirse amado; visitarse; darse consejos e información necesaria, aceptarse, etc., con fines, metas y objetivos comunes que son llevados a cabo con responsabilidad y compromiso.

Según Lin y Cols ${ }^{6}$, Stewart ${ }^{7}$ y Barrón ${ }^{5}$ algunas de las formas en que se puede estudiar el apoyo social es desde el nivel de análisis de las redes sociales, desde la perspectiva estructural, funcional y contextual, así como desde la naturaleza del apoyo social, cuando se analiza la calidad del apoyo. Así, Rodríguez ${ }^{5}$ y Buendía $^{8}$, refieren que la "red social" es aquel conjunto de personas y/o grupos que se brindan apoyo, el cual es recibido e intercambiado entre sí, en donde se mantiene el contacto entre ellos y que está conformado por una estructura en donde se llevan a cabo esas interrelaciones constituyendo una forma de cuerpo social, (familia, amigos, vecinos, compañeros de trabajo, etc.).

La importancia del apoyo social y de mantener o crear redes sociales de apoyo, es debido a que se conoce que éste genera en el individuo un desarrollo óptimo emocional y satisfactorio con consecuencias positivas hacia su salud, beneficiando su calidad de vida y a las personas que le rodean. La percepción que el sujeto tiene de que recibe apoyo, de que es confiable y efectiva, es un determinante importante de su estado de salud, 
independientemente de la condición real del sujeto 9 .

En general, es importantísimo para el individuo contar con, al menos, una persona que esté pendiente de él, que le ofrezca su ayuda tanto en los momentos felices como en los angustiantes y estresantes; así como es importante resaltar que el apoyo social se relaciona con la "mala salud" y que ésta puede agravarse o, al menos, es más probable entre los individuos que no cuentan con apoyo o el que se brinda se da de manera no adecuada.

Con lo expuesto, nos damos cuenta de lo imprescindible que es crear y mantener lazos de apoyo y mantenerlos, en cualquiera de los ámbitos donde nos encontremos, ya sea laboral o familiar. Si no contamos con alguien que nos apoye, que nos brinde su cariño, escuche, nos de algo de él, es el momento de buscarlo. Y si ya lo tenemos, habrá que cuidarlo.

\section{MATERIAL Y MÉTODO}

El estudio es descriptivo, transversal, analítico. La población de estudio incluye a los médicos familiares de cada institución. De los médicos que laboran en el seguro social se obtuvo una muestra de 154 sujetos, seleccionando al médico por un muestreo aleatorio estratificado. De los médicos que trabajan en la institución de salud y los trabajadores del Estado, por ser la población de médicos pequeña en número, se tomó a toda la población (42 médicos). En ambas instituciones la participación fue voluntaria y bajo consentimiento informado. Para recabar la información necesaria se aplicaron dos instrumentos: un cuestionario de datos generales con variables personales y laborales y un Inventario de Recursos Sociales de Díaz Veiga para evaluar el apoyo social.

Existen diferentes instrumentos que ayudan a evaluar el apoyo social. Algunos evalúan los aspectos estructurales del apoyo y otros los aspectos funcionales del mismo. Los primeros son aquellos que miden la existencia de personas como fuente de apoyo (aspectos objetivos) como por ejemplo el Social Network Index ${ }^{10}$. Los aspectos funcionales del apoyo hacen referencia a aspectos cualitativos como el grado de satisfacción con su red de apoyo (aspecto subjetivo). Por otro lado, la percepción que el sujeto tiene de que recibe apoyo y de que da resultados positivos, también puede ser medible. Para ello existen algunos instrumentos como el Instrumental-Expressive Support Scale ${ }^{6}$. Más aún, se tiene la existencia de instrumentos que evalúan ambos aspectos objetivos y subjetivos, como los es el Inventario de Recursos Sociales de Díaz Vega el cual fue utilizado en este estudio para el análisis de las redes sociales de apoyo extralaborales. Dicha escala fue construida siguiendo las recomendaciones de Satarino y Syme, validada y con índice de consistencia interna entre 35 y $86^{11,12,5}$.

El inventario original estudia el tamaño, frecuencia de contactos y tipos de apoyo con los que cuenta el sujeto sobre una escala ordinal de tres punto, además del grado de satisfacción subjetiva con su red. Incluye a la pareja, hijos, otros familiares y amigos. A partir de este instrumento se obtienen dos índices, uno referido a características objetivas de apoyo social (oscila entre 0 y 12 puntos) y otro referido a características subjetivas de satisfacción (oscila entre 0 y 3 puntos).

Para analizar las redes sociales de apoyo laborales, fue necesario agregar al cuestionario original de Díaz Veiga variables enfocadas hacia el apoyo que perciben los sujetos dentro de la institución. Tanto para los aspectos objetivos como para los subjetivos, incluyendo a compañeros de trabajo, superiores y subordinados, los cuales fueron evaluados de la misma manera que para el análisis de las redes sociales de apoyo extralaborales ${ }^{13}$.

El sistema de evaluación, los puntajes y el nivel de clasificación se describe de la siguiente manera:

1. Redes sociales extralaborales (familiares).

a)Aspectos objetivos. Se suman los aspectos objetivos de la escala (frecuencia con que ve y habla con su red de apoyo) de cada una de las interacciones (cónyuge, hijos, familiares y amigos), del cual se obtiene un puntaje y un nivel de clasificación. De 4 a 6 puntos significa que tiene una red alta, es decir que su red de 
apoyo es muy buena, lo bastante adecuada en la frecuencia con que ve y habla con sus contactos; de 7 a 9 puntos significa que tiene una red media o regular en su aspecto objetivo, y de 10 a 12 puntos que cuenta con una red de apoyo baja o mala en su frecuencia de contactos.

b) Aspectos subjetivos. Se suman los aspectos subjetivos de la escala (grado de satisfacción con red) de cada una de las interacciones (cónyuge, hijos, familiares y amigos), del cual se obtiene un puntaje y un nivel de clasificación. El mismo que para los aspectos objetivos, es decir, de 4 a 6 puntos significa que tiene una red alta, de 7 a 9 puntos red media y de 10 a 12 red de apoyo baja o mala.

\section{Redes sociales laborales.}

a)Aspectos objetivos. Se suman los aspectos objetivos de la escala (frecuencia con que ve y habla con su red de apoyo) de cada una de las interacciones (superiores, compañeros de trabajo y subordinados), de los cuales también se obtiene un puntaje y un nivel de clasificación. De 3 a 4 puntos significa que tiene una red alta, es decir que su red de apoyo es muy buena, de 5 a 7 puntos significa que tiene una red media y de 8 a 9 puntos se considera una red de apoyo baja.

b)Aspectos subjetivos. Se suman los aspectos subjetivos de la escala (grado de satisfacción con red) de cada una de las interacciones (superiores, compañeros de trabajo y subordinados), del cual se obtiene un puntaje y un nivel de clasificación iguales que para las redes objetivas laborales.

Al final se realizó una valoración total: la suma de los puntajes de ambos aspectos objetivos y para los subjetivos, que se sitúan en un nivel de clasificación con el propósito de identificar si toda la red de apoyo con la que cuenta el sujeto es alta, media o regular.

\section{RESULTADOS}

Participaron 196 médicos, de los cuales 42 trabajan para la institución que labora para los trabajadores del Estado y 154 pertenecen a la que brinda servicios a la población derechohabiente del seguro social. Setenta y tres sujetos $(37,1 \%)$ son mujeres y $124(62.9 \%)$ hombres. La edad mínima reportada fue de 25 años y la mayor de 62 . El promedio de edad fue de 46,29 años. La mayoría son casados 153 (79,7\%). El nivel máximo de estudios es la especialidad $(92,9 \%)$, los que no cuentan con ella, que sólo son médicos cirujanos y parteros, son considerados por parte de su contrato de

Tabla 1: Distribución de la población en número y porcentaje según aspectos objetivos (frecuencia de contactos familiar y laboral) en cada una de las instituciones de estudio

\begin{tabular}{|c|c|c|c|c|c|c|c|c|}
\hline \multirow[t]{3}{*}{$\begin{array}{l}\text { Nivel de } \\
\text { calificación }\end{array}$} & \multicolumn{8}{|c|}{$\begin{array}{l}\text { Escala de evaluación de apoyo y contactos sociales de Díaz Veiga } \\
\text { modificada por Aranda y Pando, } 2004 .\end{array}$} \\
\hline & \multicolumn{4}{|c|}{ Apoyo extralaboral } & \multicolumn{4}{|c|}{\begin{tabular}{|c|} 
Apoyo laboral \\
\end{tabular}} \\
\hline & \multicolumn{2}{|c|}{$\begin{array}{l}\text { Aspectos objetivos } \\
\text { (frecuencia de contactos) } \\
\text { (Trabajadores del Estado) }\end{array}$} & \multicolumn{2}{|c|}{\begin{tabular}{l|} 
Aspectos objetivos \\
(frecuencia de contactos) \\
(Seguro social)
\end{tabular}} & \multicolumn{2}{|c|}{$\begin{array}{c}\text { Aspectos objetivos } \\
\text { (frecuencia de contactos) } \\
\text { (Trabajadores del Estado) }\end{array}$} & \multicolumn{2}{|c|}{$\begin{array}{l}\text { Aspectos objetivos } \\
\text { (frecuencia de contactos) } \\
\text { (Seguro social) }\end{array}$} \\
\hline $\begin{array}{l}\text { Red alta } \\
\text { (muy buena } \\
\text { red de apoyo) }\end{array}$ & $\begin{array}{l}\mathrm{N}^{\circ} \text {. } \\
39\end{array}$ & $\begin{array}{c}\% \\
92,9\end{array}$ & $\begin{array}{l}\mathrm{N}^{\mathrm{o}} \text {. } \\
122\end{array}$ & $\begin{array}{l}\% \\
79,2\end{array}$ & $\begin{array}{r}\mathrm{N}^{\mathrm{o}} \text {. } \\
32\end{array}$ & $\begin{array}{l}\% \\
76,2\end{array}$ & $\begin{array}{l}N^{\circ} \text {. } \\
112\end{array}$ & $\begin{array}{c}\% \\
72,7\end{array}$ \\
\hline $\begin{array}{l}\text { Red media } \\
\text { (regular red } \\
\text { de apoyo) }\end{array}$ & 3 & 7,1 & 31 & 20,1 & 10 & 23,8 & 40 & 26,0 \\
\hline $\begin{array}{l}\text { Red baja } \\
\text { (mala red de } \\
\text { apoyo) }\end{array}$ & 0 & 0 & 1 & 0,6 & 0 & 0 & 2 & 13 \\
\hline Totales & 42 & 100 & 154 & 100 & 42 & 100 & 154 & 100 \\
\hline
\end{tabular}

Fuente: directa 
trabajo como médicos familiares. De los 196 médicos, 183 (93,4\%), 4 son de base, eventuales y 9 residentes (especialidad de medicina familiar). El tiempo menor de labor continua como médico familiar es de 6 meses sólo una persona rebasa los 30 años, el promedio fue de 16,47 años, el $25,9 \%$ (51 personas) tienen entre 10 y 14 años de laborar en ese puesto, siendo el turno matutino el de mayor población $104(52,8 \%)$ seguido por el vespertino $82(41,6 \%) .107$ de los médicos $(54,6 \%)$ manifiestan trabajar 30 horas a la semana mientras que cincuenta y cuatro $(27,6 \%)$ refieren hacerlo por 40 horas.

Como se observa en la Tabla 1, las redes sociales de apoyo extralaborales y laborales en ambas instituciones y en su aspecto objetivo, es decir en cuanto a la frecuencia de contacto con su red de apoyo, son consideradas "muy buenas", si partimos de los criterios antes expuestos.

Llama la atención que al igual que para los aspectos objetivos (frecuencia de contacto), en los subjetivos, la mayoría de los médicos la consideran "muy buena". resultados similares para las dos instituciones (Tabla 2). observamos en la tabla 4 para los médicos familiares del seguro social.

Tratando de concentrar la información, en la Tabla 5, se puede observar toda la información acerca de la red de apoyo en ambas instituciones, tomando en cuenta ambos aspectos objetivos y subjetivos, así como el apoyo extralaboral y el laboral, calificando su red como "muy buena".

En cuanto al análisis inferencial realizado entre las variables sociodemográficas y laborales como edad, sexo, estado civil, escolaridad, antigüedad en la institución y en el puesto actual, no se obtuvieron asociaciones ni factores de riesgo significativos, debido probablemente a que el apoyo social resultó ser "muy bueno".

En general, llama la atención que las redes de apoyo en ambas instituciones son muy buenas en relación a la frecuencia de contactos y a la satisfacción que sienten y perciben de su red de apoyo ya sea familiar o extralaboral como en la laboral. Al comparar las redes de apoyo entre las instituciones, se observa que en todas las tablas, los porcentajes mayores se inclinan hacia el contar con una red alta o muy buena, cifras menores hacia

Tabla 2: Distribución de la población en número y porcentaje según aspectos subjetivos (grado de satisfacción con su red familiar y laboral), en cada una de las instituciones de estudio

\begin{tabular}{|c|c|c|c|c|c|c|c|c|}
\hline \multirow[t]{4}{*}{$\begin{array}{l}\text { Nivel de } \\
\text { calificación }\end{array}$} & \multicolumn{8}{|c|}{$\begin{array}{l}\text { Escala de evaluación de apoyo y contactos sociales de Díaz Veiga } \\
\text { modificada por Aranda y Pando, } 2004 .\end{array}$} \\
\hline & \multicolumn{4}{|c|}{ Apoyo extralaboral } & \multicolumn{4}{|c|}{ Apoyo laboral } \\
\hline & \multicolumn{2}{|c|}{$\begin{array}{l}\text { Aspectos subjetivos } \\
\text { (Trabajadores del Estado) }\end{array}$} & \multicolumn{2}{|c|}{$\begin{array}{l}\text { Aspectos subjetivos } \\
\text { (Seguro social) }\end{array}$} & \multicolumn{2}{|c|}{$\begin{array}{l}\text { Aspectos subjetivos } \\
\text { (Trabajadores del Estado) }\end{array}$} & \multicolumn{2}{|c|}{$\begin{array}{l}\text { Aspectos subjetivos } \\
\text { (Seguro social) }\end{array}$} \\
\hline & $\mathrm{N}^{\mathrm{o}}$ & $\%$ & $\mathrm{~N}^{\mathrm{o}}$ & $\%$ & $\mathrm{~N}^{\mathrm{o}}$. & $\%$ & $\mathrm{~N}^{\mathrm{o}}$. & $\%$ \\
\hline Red alta & 40 & 952 & 132 & 85,7 & 31 & 73,8 & 79 & 51,3 \\
\hline Red media & 1 & 2,4 & 21 & 12,3 & 10 & 23,8 & 69 & 44,8 \\
\hline Red baja & 1 & 2,4 & 1 & 2.0 & 1 & 2.4 & 6 & 3.9 \\
\hline Totales & 42 & 100 & 153 & 100 & 42 & 100 & 154 & 100 \\
\hline
\end{tabular}

Fuente: directa

Continuando con el análisis estadístico, los resultados en cuanto al apoyo social que reportaron los médicos familiares pertenecientes a la Institución para los Trabajadores del Estado, refieren contar con una "muy buena" red de apoyo tanto en la frecuencia de contactos con su red de apoyo como con el grado de satisfacción con la misma (Tabla 3), al igual lo contar con una red media o regular, y muy pocos médicos refieren no contar con apoyo.

Se hace necesario recalcar que sobre la base de estos resultados, los análisis inferenciales no mostraron asociación alguna entre las variables, pero sí que los médicos cuentan con un adecuado apoyo social. 
Tabla 3: Distribución de la población en número y porcentaje de ambos aspectos en los médicos generales (familiares) para los trabajadores del Estado

\begin{tabular}{|l|c|c|c|c|}
\hline \multirow{2}{*}{$\begin{array}{l}\text { Nivel de } \\
\text { calificación }\end{array}$} & \multicolumn{3}{|c|}{$\begin{array}{c}\text { Escala de evaluación de apoyo y contactos } \\
\text { sociales de Díaz Veiga modificada por } \\
\text { Aranda y Pando, 2004. }\end{array}$} \\
\hline & \multicolumn{2}{|c|}{ Apoyo objetivos } & \multicolumn{2}{c|}{ Apoyo subjetivos } \\
\cline { 2 - 5 } & No. & $\%$ & No. & $\%$ \\
\hline Red alta & 37 & 88,1 & 35 & 8,3 \\
\hline Red media & 5 & 11,9 & 7 & 16,7 \\
\hline Red baja & 0 & 0 & 0 & 0 \\
\hline Totales & 42 & 100 & 42 & 100 \\
\hline
\end{tabular}

Fuente: directa

Tabla 4: Distribución de la población en número y porcentaje de ambos aspectos en los médicos familiares del seguro social

\begin{tabular}{|l|c|c|c|c|}
\hline \multirow{2}{*}{$\begin{array}{l}\text { Nivel de } \\
\text { calificación }\end{array}$} & \multicolumn{3}{|c|}{$\begin{array}{c}\text { Escala de evaluación de apoyo y contactos } \\
\text { sociales de Díaz Veiga modificada por } \\
\text { Aranda y Pando, 2004. }\end{array}$} \\
\hline & \multicolumn{2}{|c|}{ Apoyo objetivos } & \multicolumn{2}{c|}{ Apoyo subjetivos } \\
\cline { 2 - 5 } & No. & $\%$ & No. & $\%$ \\
\hline Red alta & 128 & 83,1 & 114 & 74,0 \\
\hline Red media & 24 & 15,6 & 38 & 24,7 \\
\hline Red baja & 2 & 1,3 & 2 & 1,3 \\
\hline & 155 & 100 & 154 & 100 \\
\hline
\end{tabular}

Fuente: directa

Tabla 5: Distribución de la población en número y porcentaje en ambos aspectos objetivos y subjetivos y por toda la población en estudio.

\begin{tabular}{|l|c|c|c|c|}
\hline \multirow{2}{*}{$\begin{array}{l}\text { Nivel de } \\
\text { calificación }\end{array}$} & \multicolumn{4}{|c|}{$\begin{array}{c}\text { Escala de evaluación de apoyo y contactos } \\
\text { sociales de Díaz Veiga modificada por } \\
\text { Aranda y Pando, 2004. }\end{array}$} \\
\hline \multirow{2}{*}{ Red alta } & \multicolumn{3}{|c|}{$\begin{array}{c}\text { Apoyo extralaboral } \\
\text { Aspectos objetivos } \\
\text { (ambas instituciones) }\end{array}$} & \multicolumn{2}{|c|}{$\begin{array}{c}\text { Apoyo laboral } \\
\text { Aspectos subjetivos } \\
\text { (ambas instituciones) }\end{array}$} \\
\cline { 2 - 5 } & No. & $\%$ & No. & $\%$ \\
\hline Red media & 165 & 84.2 & 149 & 76.0 \\
\hline Red baja & 29 & 14.8 & 45 & 23.0 \\
\hline Totales & 2 & 1.0 & 2 & 1.0 \\
\hline & 196 & 100 & 196 & 100 \\
\hline
\end{tabular}

Fuente: directa

\section{DISCUSIÓN}

Pensemos en la red social como un procesode construcción permanente tanto singular como colectivo. Cada individuo que conforma la red de apoyo se enriquece a través de las múltiples y variadas relaciones que se llevan entre sí.

El apoyo social laboral o familiar, aparece en los estudios como una de las principales variables que permiten prevenir y aliviar las respuestas que el individuo genera ante los acontecimientos a los que se enfrenta y afronta. Es así como el apoyo social juega un papel importante en todas las personas, al suponer que un buen apoyo social proporciona mejores niveles de salud y bienestar tanto físico, como biológico, psicológico y social y viceversa, un buen estado de salud en estas esferas facilitaría el mantener un buen apoyo social.

En el presente estudio, dado que las redes sociales laborales y las familiares resultaron dentro de su clasificación "muy buenas", los análisis de asociación no tuvieron significancia alguna.

Sin embargo, se hace necesario mencionar la presencia de estudios en donde el apoyo social ha disminuido o prevenido el riesgo de desarrollar la enfermedad. Como en individuos que padecen enfermedades cardiovasculares, cáncer o enfermedades crónico-degenerativas. También en personas en etapa terminal o con trastornos psicopatológicos como depresión, ansiedad, trastornos de conducta y del sueño, ante el estado civil, el género y hasta en la jubilación ${ }^{4,13-17}$.

Son pocos los trabajos que hablan sobre esta variables "apoyo social", por lo tanto el tipo de población en las cuales se han estudiado no son tan variadas. Aún así tenemos por ejemplo el estudio de Aranda ${ }^{18}$ en personal docente y administrativo que labora en un centro de educación superior, con resultados contrarios a los encontrados con los médicos. El nivel de calificación encontrado fue bajo, tanto en la frecuencia de contacto como en el grado de satisfacción con su red; sin embargo, si se encontraron variables con asociación significativa: el apoyo extralaboral o familiar 
con la edad, el estado civil, el contar con hijos y el apoyo laboral, con el estado civil y el turno de trabajo.

Es preocupante darse cuenta de cómo se presenta el apoyo social en la población adulta mayor. La misma autora, realizó un estudio en población de la tercera edad jubilada de la zona metropolitana de Guadalajara, donde se obtuvieron datos alarmantes: el apoyo social es nulo y malo, sin embargo, las personas decían sentirse satisfechas con ese poco e inadecuado apoyo, claro está que se habla del apoyo familiar o extralaboral ${ }^{19}$.

Es imprescindible estar a pendiente sobre el tipo de ayuda que reciben y perciben los individuos, así como quienes son las personas que forman su red de apoyo. No olvidemos que el apoyo social y la salud se relacionan entre sí y que la "mala salud" es más pronunciada y más probable entre aquellos a los que les falta apoyo o carecen de él.

Ante esto, concluimos que es necesario crear y mantener lazos con las personas que nos rodean, tanto en el ámbito laboral como en el familiar.

Es urgente poner los pies en la tierra y observar nuestro alrededor y si nos damos cuenta que estamos solos, habrá que buscar a ese alguien que será nuestro apoyo y, si ya lo tenemos habrá que cuidarlo para no perderlo.

\section{REFERENCIAS}

1. HOUSE, J.S. Work stress and social support. Addison-Weslwey 1981.

2. THOITS, P.A. Conceptual, methodological, and theoretical problems in studying social support as a buffer against loife stress. Journal of Health and Social Behavior 1982: 23:145-149.

3. FELTON BJ, SHINN M. Social integration and social support: Moving social support beyond the individual level. Journal of Community Psychology. Num.20,1992:103-115.

4. RODRÍGUEZ MARÍN JESÚS. Psicología Social de la Salud. Edit. Síntesis, Madrid, 1995.

5. BARRÓN A. Apoyo social. Aspectos teóricos y aplicaciones. Ed. Siglo XXI de España editores, Madrid, 1996.
6. LIN N, DEAN A, ENSEL WM. (1986). Social support, life events and depressions, Nueva York, Academic Press. En Barrón A. Apoyo social. Aspectos teóricos y aplicaciones. De. Siglo XXI de España editores, Madrid, 1996.

7. STEWART MJ. Soccial Support Instrument Created By Nurse Investigators. Nursing Research, 1989;38(5): 268-275.

8. BUENDÍA J. Envejecimiento y psicología de la salud. Edit. Siglo Veintiuno de España S.A. España, 1994.

9. HELLER K., LAKEY B. (1985). Percived support and social interaction among friends and confidants: En Barrón A. Apoyo social. Aspectos teóricos y aplicaciones. Ed. Siglo XXI de España editores, Madrid, 1996.

10. 10. BERKMAN L.F., SYME S.L. Social networks, host resistance and mortality: A nine year follow-up study of Alameda Country residents. American Journal of Epidemiology 1985;109:186-204.

11. 11. MONTORIO IGNACIO. Evaluación psicológica en la vejez: Instrumentación desde un enfoque multidimensional. Tesis doctoral no publicada. Universidad Autónoma de Madrid, 1990.

12. 12. MONTORIO IGNACIO. La persona mayor. Guía aplicada de evaluación psicológica. Edit. Ministerio de asuntos sociales. Instituto Nacional de Servicios Sociales. $1^{\mathrm{a}}$ edición, Madrid 1994.

13. 13. ARANDA BELTRÁN C. Apoyo social y Síndrome de quemarse en el trabajo"burnout" en los médicos familiares de base del Instituto Mexicano del Seguro Social, Zona Metropolitana de Guadalajara. Tesis Doctoral en Ciencias de la Salud Pública, Enero 2004.

14. 14. CHAPPELL NL, SEGALL A, LEWIS DG.(1990). Gender and Helping Networks among Day Hospital and Senior Centre Participants. Canadian Journal on Aging. Num 9(3):220-233. En Buendía José. Envejecimiento y psicología de la salud. Edit. Siglo Veintiuno de España S.A. España, 1994.

15. 15. MUTRAN EJ,PHD; DANIS M,MD,PHD, BRATTON KA, PhD, Sudha S, PhD, and Hanson L, MD. Attitudes of the critically III toward prolonging life: The role of social support. The Gerontological Society of América. The Gerontologist. 1997, Vol.37,No.2,192-199.

16. MUTCHLER JAN E., BURR JEFFREY A., MASSAGLI MICHAEL P., PIENTA AMY. Work transitions and health in later life. The Journals of Gerontology; Washington 1999; vol 54: 252-261

17. ARANDA BC, PANDO MM, SALAZAR EJG, TORRES LTM, ALDRETE RMG, PÉREZ RMB. (2005a). Síndrome de burnout y manifestaciones clínicas en los médicos familiares del Instituto Mexicano del Seguro Social, Guadalajara, México. Revista Cubana de Salud Pública 31(2). 
18. ARANDA BC, PANDO MM, SALAZAR EJ. Situación del apoyo social en el personal docente y administrativo que labora para un Centro de Educación Superior, Universidad de Guadalajara, México. Populus per saluten 2005;1(2-3):13-15.
19. ARANDA BC, PANDO MM. Redes sociales de apoyo en el adulto mayor jubilado de Guadalajara, Jal. México. Humanitatis 2004; 2(4-5):76-80. 